\title{
Análisis estadístico textual de políticas de financiamiento de la educación superior: evidencia en países de la Organización para la Cooperación y el Desarrollo Económico (OCDE)
}

\author{
María I Barbosa-Camargo', Suelen E Castiblanco-Moreno e Iván F Medina-Arboleda² \\ (1) Facultad de Ciencias Económicas y Sociales, Universidad de La Salle, Bogotá-Colombia. \\ (correo-e: mibarbosa@unisalle.edu.co; secastiblanco@unisalle.edu.co) \\ (2) Facultad de Psicología, Universidad Católica de Colombia, Bogotá-Colombia. (correo-e: ifmedina@ucatolica.edu.co)
}

Recibido Jun. 23, 2020; Aceptado Ago. 20, 2020; Versión final Sep. 23, 2020, Publicado Feb. 2021

\section{Resumen}

El objetivo de este estudio es validar empíricamente si el planteamiento de la Organización para la Cooperación y el Desarrollo Económico (OCDE) respecto a los modelos de financiamiento de educación superior se corresponde con el contenido de las políticas de financiamiento declaradas por países miembros. La OCDE plantea que los modelos implementados por los países se pueden clasificar en cuatro tipos, dada la relación entre tasas de matrícula y el sistema de incentivos a estudiantes. Se construye un modelo multifactorial a partir de datos textuales para analizar los corpus de las políticas de financiamiento de la educación superior de cuatro países: Noruega, Alemania, Inglaterra y Colombia. Los resultados muestran que el análisis factorial da cuenta del $75.6 \%$ de la varianza a partir de los primeros dos ejes: "financiamiento oferta y demanda" (40.9\%) y "costos para los estudiantes" (34.6\%). Se concluye que el sistema planteado por la OCDE es funcional para describir los modelos de financiamiento de los países analizados.

\section{Textual statistical analysis of higher education policy financing: evidence from member countries of the Organization for Economic Cooperation and Development (OECD)}

\begin{abstract}
The primary objective of the present study is to empirically validate whether higher education financing models proposed by the Organization for Economic Cooperation and Development (OECD) is in agreement with financing policies applied by member countries. The OECD states that financing models implemented by member countries can be classified into four different types depending on the relationship between enrollment rates and student incentive systems. A multifactorial model is constructed from textual data to analyze the bodies of higher education financing policies in four countries: Norway, Germany, the United Kingdom, and Colombia. The results show that factorial analysis explains $75.6 \%$ of variance, based on the first two axes: "supply and demand financing" (40.9\%) and "costs for students" (34.6\%). It is concluded that the financing system proposed by the OECD works to describe the financing models of the countries examined.
\end{abstract}




\section{INTRODUCCIÓN}

Tanto la importancia de la educación superior, como los aspectos relacionados con su financiación, han sido ampliamente discutidos por diversos investigadores sociales. Una de las posturas, la económica, ha centrado la discusión en la dualidad entre bien público y bien privado, que deriva en la identificación de los roles y objetivos otorgados a la educación en la sociedad, y con ello, en la financiación compartida de recursos públicos y privados. Según cifras de la Organización para la Cooperación y el Desarrollo Económico (OECD, 2019), entre 2005 y 2016 el gasto promedio en instituciones de educación superior en los países miembros se incrementó en un $28 \%$, siendo más del $30 \%$ financiado por fuentes privadas. Asimismo, los costos de matrícula se han incrementado en más del $20 \%$ entre 2007 y 2017 , y han sido las fuentes primarias las llamadas a contribuir en mayor proporción con el aumento o introducción de los costos de matrícula.

En este contexto, los recursos adicionales son colocados alrededor del mundo buscando incrementar el acceso a la educación superior al tiempo que aumentan las expectativas sobre sus resultados. En efecto, el número global de estudiantes que persiguen la educación superior ha crecido continuamente sobre las dos décadas pasadas, empero, la expansión sólo es sostenible si existe un balance entre la oferta de graduados y las necesidades del mercado laboral (OECD, 2018). Después de todo, los resultados de la educación superior se encuentran encaminados principalmente a conducir transformaciones sociales y atender necesidades del mercado laboral, en cuanto su misión primaria es el desarrollo y transferencia del conocimiento; lo que ha generado una ampliación de oportunidades de aprendizaje que, a su vez, se ha visto acompañada de una inevitable transformación de un sistema de élite a un sistema de masas. Al respecto, las estadísticas destacan el aumento sostenido en educación terciaria en el mundo, que pasó del $24 \%$ en 2005 al 30\% en 2010 y al 38\% en 2018; para Colombia estos valores han sido del 30, el 39 y el 55\% respectivamente (Unesco, 2020).

En general, los países han respondido a estos retos de expandir la educación superior y los mecanismos de soporte financiero han aliviado algunas de las necesidades de estudios adicionales, con la promesa de altos retornos y opciones de reembolso flexibles, desde visiones más holísticas en las que confluyen otros agentes como empleadores, industria e instituciones, las cuales logran un balance entre un gran número de matriculados con la necesidad de cubrir costos y mantener la relevancia y calidad de los programas (OECD, 2019). Sin embargo, no todos los países han respondido de la misma manera. Más aún, la diversidad de sistemas educativos implementados son consecuencia tanto de una decisión económica como de una decisión política, usualmente tomada frente a la propia capacidad para aumentar la competencia en el mercado o de expandir los elementos de no exclusión y no rivalidad en la producción y distribución de la educación superior (Marginson, 2007); lo que lleva a dos distinciones: la política, entre estado y no estado; y la económica, entre mercado y no mercado (Marginson, 2018).

Al final, lo cierto de este debate es que la forma elegida para financiar la educación afecta tanto la cantidad como la calidad de la misma, e igualmente influye en cómo se distribuyen las oportunidades educativas en la sociedad. Diversos autores se han concentrado en los costos educativos que deben asumir los estudiantes, entre otras por la afectación negativa al desempeño académico (Gaxiola, Gaxiola, Corral y Escobedo, 2020), así como en la magnitud de los subsidios proporcionados por la sociedad (De Donder y Martínez-Mora, 2017; Del Rey y López-García, in press); la ineficiencia del mercado en la provisión de créditos y el rol del Estado en la financiación de la educación superior (Van-Long, 2019) o la evolución del gasto público y la sostenibilidad de los subsidios (Haupt, 2012).

También se encuentran trabajos acerca de las particularidades de los mecanismos de financiación: entre préstamos de ingreso contingente y los tradicionales dependiendo del contexto (Chapman y Doris, 2019); o en la colocación de recursos basada en el desempeño o el resultado de las instituciones (Adam, 2020), esta última analizada incluso en contextos particulares como Chile (Araneda-Guirriman y Pedraja-Rejas, 2016) y frente a su influencia en la autonomía de las universidades (Araneda-Guirriman, Gairín-Sallán y PedrajaRejas, 2018). Pero estas no son las únicas aproximaciones al tema realizadas. Uno de los modelos de financiación de educación superior pioneros, llamado triángulo de la coordinación, es propuesto por Clark (1983) (Citado en Jongbloed, 2003), quien concibe la educación superior como un sistema que tiende a resolver la contraposición de los intereses del Estado, el Mercado y las Instituciones de Educación Superior, al constituir tres grandes grupos de poder y de presión que intervienen en la toma de decisiones del sistema. De acuerdo con esta propuesta, según el rol de cada actor, existirían tres tipos de modelos, i) el burocrático, donde las autoridades gubernamentales deciden sobre la prestación del servicio educativo; ii) el de mercado, en el que las universidades obtienen recursos de la venta de servicios educativos demandados por los usuarios; y, iii) el colegiado, en el que las instituciones tienen mayor autonomía para distribuir los recursos recibidos del Estado y del resto de la sociedad. 
Estos planteamientos han llevado a que simultáneamente, se desarrolle un debate en torno a los costos educativos, que pueden ser compartidos o asumidos bajo esquemas de gratuidad. La razón de ser del debate se encuentra en el trade off entre eficiencia y equidad en escenarios donde, por una parte, se favorece la gratuidad o la disminución del costo de matrícula pagado por el estudiante, y por otra, los recursos públicos son canalizados hacia el otorgamiento de becas y créditos que pueden tener efectos importantes en personas de menores recursos (Gershenfeld, Zhan y Hood, 2019; Río-Ruíz, Jiménez-Rodrigo y Caro-Cabrera, 2015).

Si bien, hay muchos países desarrollados en los que el sistema contempla gratuidad en los costos directos, los padres o los mismos estudiantes perciben grandes costos asociados al sostenimiento que podrían ser compartidos. Bajo esta perspectiva, la financiación es compartida entre cinco agentes: aquellos que pagan impuestos, el estudiante y su familia quienes pagan la matrícula y asumen costos de sostenimiento, entidades financieras que ofrecen préstamos o ayudas financieras y, por último, las instituciones educativas o donantes privados que pueden ofrecer ayudas a estudiantes que requieran apoyo en la financiación (Johnstone, 2004; Sanyal y Johnstone, 2011). Sin embargo, dicha estructura de costos compartidos no resulta bienvenida si los estudiantes no pueden pagar su parte (Van Long, 2019). En particular, cuatro modelos pueden ser planteados al combinar las tasas de matrícula y el sistema de incentivos otorgados a los estudiantes. Este esquema es el utilizado por la OECD, en cuanto refleja tanto la importancia que otorgan los países al valor social de la educación como la dependencia de las instituciones educativas de recursos estatales, la eficiencia de modelos con costos compartidos o las dificultades que estos pueden presentar (OECD, 2019).

Tabla 1. Tasas de matrícula y sistemas de incentivos. (Adaptado de OECD, 2019)

\begin{tabular}{|c|c|c|}
\hline & Generosos programas de apoyo a estudiantes & Programas de apoyo a estudiantes en desarrollo \\
\hline \multirow[t]{3}{*}{$\begin{array}{l}\text { Sin o con } \\
\text { bajas tasas } \\
\text { de } \\
\text { matrícula }\end{array}$} & $\begin{array}{l}\text { Hay bajas o ninguna tasa de matrícula en un } \\
\text { sistema de incentivos generoso, financiado con } \\
\text { estructuras de impuestos progresivas y altas tasas } \\
\text { de impuestos sobre el ingreso. }\end{array}$ & $\begin{array}{l}\text { Los estudiantes pagan bajas tasas de matrícula } \\
\text { con un sistema de incentivos subdesarrollado, } \\
\text { donde las instituciones educativas dependen en } \\
\text { gran medida de recursos estatales y los } \\
\text { estudiantes y sus familias asumen los costos de } \\
\text { sostenimiento. }\end{array}$ \\
\hline & $\begin{array}{l}\text { El modelo refleja la importancia que se otorga al } \\
\text { valor social de la educación tales como igualdad de } \\
\text { oportunidades y equidad social, basados en el } \\
\text { principio que acceder a la educación es un derecho } \\
\text { más que un privilegio. }\end{array}$ & $\begin{array}{l}\text { Lo conforman países europeos: Austria, Bélgica, } \\
\text { Francia, Alemania, Italia y Suiza, República Checa, } \\
\text { Irlanda, Polonia, Portugal y España. }\end{array}$ \\
\hline & $\begin{array}{l}\text { Está compuesto por los países nórdicos: } \\
\text { Dinamarca, Finlandia, Noruega y Suecia. Más del } \\
55 \% \text { de estudiantes se beneficia de becas o } \\
\text { créditos o ambos otorgados por el gobierno. }\end{array}$ & \\
\hline \multirow[t]{2}{*}{$\begin{array}{l}\text { Altas tasas } \\
\text { de } \\
\text { matrícula }\end{array}$} & $\begin{array}{l}\text { Los estudiantes tienen altos costos de matrícula, lo } \\
\text { que genera potenciales barreras a la entrada a la } \\
\text { educación terciaria, al mismo tiempo que tienen la } \\
\text { oportunidad de tener incentivos importantes por } \\
\text { parte de sus gobiernos en un esquema de costos } \\
\text { compartidos entre el gobierno, los hogares y las } \\
\text { entidades privadas sobre la base de que contar } \\
\text { con recursos privados amplia los recursos totales } \\
\text { para la educación. }\end{array}$ & \multirow[t]{2}{*}{$\begin{array}{l}\text { Los estudiantes pagan tasas de matrícula elevadas } \\
\text { en un sistema de incentivos a estudiantes } \\
\text { subdesarrollado; lo que genera dificultades en los } \\
\text { estudiantes para financiar los estudios. Hacen } \\
\text { parte de este grupo países como Chile, Colombia, } \\
\text { Japón y Corea. }\end{array}$} \\
\hline & $\begin{array}{l}\text { Pertenecen a este grupo Australia, Canadá, Nueva } \\
\text { Zelanda, Inglaterra y Estados Unidos de América. }\end{array}$ & \\
\hline
\end{tabular}

En este marco, la presente investigación compara las políticas de educación superior de cuatro países (Noruega, Alemania, Inglaterra y Colombia), tomados como ejemplo de cada uno de los modelos descritos en el esquema utilizado por la OECD (2019), para de esta manera contrastar el discurso establecido desde su política educativa frente al valor social y la eficiencia del modelo de financiación adoptado. Se considera que la contribución de la investigación se da en varios sentidos. En primer lugar, se aporta evidencia al debate entre eficiencia y equidad en la adopción de los modelos de financiación de educación superior, y frente a la discusión relacionada con sí la decisión política está alineada con la económica. En segundo lugar, se propone una metodología alternativa, al utilizar un análisis estadístico de datos textuales, que permite una aproximación al modelo de financiamiento sobre la base de la política educativa oficial. En tercer lugar, se presenta una caracterización de los modelos que muestra las diferencias obtenidas en su implementación, basados en indicadores de acceso como la tasa bruta de matrícula y de los recursos destinados a la educación superior, que dejan al descubierto ciertas disparidades. 
El documento está estructurado en cinco secciones, incluyendo esta introducción. La segunda sección presenta el método implementado, que describe brevemente en qué consiste el análisis estadístico de datos textuales (AEDT). Seguidamente, se encuentra la sección de resultados, que incluye una caracterización estadística a partir de indicadores de los modelos de financiación de cada país junto con los resultados del método AEDT. Las últimas secciones discuten y concluyen.

\section{METODOLOGIA}

Las técnicas multivariantes de estudio de datos textuales, como el análisis factorial de correspondencias y el análisis de clusters permiten la aproximación a grandes cuerpos de datos textuales, de forma tal que, a través de la articulación entre la lingüística matemática, la lexicología y la estadística, sea posible interpretar el sentido de los textos (Beaudouin, 2016; Romero-Pérez, Alarcón-Vásquez, García-Jiménez, 2018). Así pues, el Análisis Estadístico de Datos Textuales se fundamenta en el recuento de unidades textuales previamente definidas, en las que a través del Análisis Factorial de Correspondencias de tablas léxicas se establece la magnitud de la asociación y similitud - diferencia de las palabras presentes en un texto o discurso (Beaudouin, 2016; Gentzkow, Lelly y Taddy, 2019). El estudio analizó las muestras textuales con el software -Système Portable pour l'Analyse de Donne -SPAD Versión (9.1).

\section{Corpus textual}

El corpus analizado corresponde a lo que Borja-Orozco et al (2008) denominan como corpus informatizado "una colección de textos seleccionados según criterios lingüísticos codificados de modo estándar y homogéneo, con la finalidad de ser tratados mediante procesos informáticos y destinados a reflejar su comportamiento" (p. 574). En este estudio se analizan documentos que describen la política de financiamiento de la educación superior de cuatro países, cada uno de ellos representativos de los cuatro modelos del esquema utilizado por la OECD, con el propósito de establecer el nivel de concordancia entre sus discursos (políticas) y el modelo al que la OECD los asigna. Los cuatro países seleccionados fueron: Noruega, como representante del Modelo 1: bajos costos de matrícula y generosos programas de apoyo; Alemania, como representante del Modelo 2: bajos costos de matrícula y programas de apoyo; Inglaterra, como ejemplar del Modelo 3: altos costos de matrícula y generosos programas de apoyo; y Colombia, como muestra del Modelo 4: altos costos de matrícula y programas de apoyo.

El documento que describe el modelo de financiamiento de la educación superior en Noruega es el "Act Relating to student finantial aid" (Acta de ayuda financiera para los estudiantes) del Ministerio de Educación e Investigación. En esta acta se presenta el propósito y alcance de la política de financiamiento de la educación superior en Noruega. Para el caso de Alemania, se seleccionó el estudio sobre su sistema de financiamiento realizado por la OECD en el año 2006. En este documento, se sintetizan las características del modelo de financiación, así como la relación entre los diferentes actores del sistema de educación superior.

Para ejemplificar el modelo 3, se eligió el documento que describe el más reciente esquema de financiamiento y apoyo a estudiantes en Inglaterra. Este texto describe la política de financiamiento y sus cambios más significativos con respecto al sistema previo. Finalmente, para el caso colombiano, se seleccionó el "Acuerdo por lo superior 2034". Propuesta de política pública para la excelencia de la educación superior en Colombia en el escenario de la paz". Este documento cuenta con varias secciones, por lo que, con el propósito de la comparabilidad de los corpus, se seleccionó solo el apartado de financiación. Atendiendo a un criterio de comparabilidad y, dado que, el análisis es de carácter textual, los documentos se analizaron en inglés, idioma original de tres de los cuatro documentos analizados para evitar la pérdida de información en la traducción.

\section{Procedimiento y umbral elegido}

El corpus inicial del que partió el análisis contaba con un total de 9973 palabras; posteriormente, se eliminaron las palabras con longitud 1, frecuencia 1 y preposiciones, como resultado de esta eliminación el corpus definitivo fue de 7444 palabras, equivalente al $75 \%$ del corpus original. De estas 7444 palabras, 778 corresponden a palabras diferentes.

Tabla 2. Vocabulario de palabras

\begin{tabular}{|l|c|}
\hline & Vocabulario de palabras \\
\hline Número total de textos & 4 \\
\hline Número original de palabras & 9973 \\
\hline Número final de palabras & 7444 \\
\hline Número de palabras distintas & 778 \\
\hline Porcentaje de palabras distintas & 10.45 \\
\hline
\end{tabular}




\section{Análisis de datos}

El análisis de los documentos de política pública se realizó en el software SPAD®. V. 9.1 para análisis de datos textuales. Se analizaron las frecuencias de uso de las palabras, y se implementó un análisis de correspondencias para estimar las palabras características de cada documento ejemplar de los diferentes modelos de financiamiento descritos en el apartado teórico.

\section{RESULTADOS}

Esta sección se organiza en dos partes. En primer lugar, se caracteriza el funcionamiento de los diferentes modelos de financiamiento. En segundo lugar, se muestran los resultados de los análisis de frecuencia de palabras de cada modelo y el plano factorial resultante del análisis de correspondencias.

\section{Contextualización de modelos de financiación}

Siguiendo la revisión de literatura y los modelos de financiamiento presentados en la introducción, la tabla 3 reúne las principales características de los modelos de financiación de los países seleccionados.

Tabla 3. Comparación modelos de financiación entre países. (Adaptado de OECD, 2019)

\begin{tabular}{|c|c|c|c|}
\hline País & Modelos de financiación & Instrumentos de financiación & $\begin{array}{c}\text { Tasa Bruta de } \\
\text { Matriculación, } 2017\end{array}$ \\
\hline \multirow[t]{5}{*}{ Noruega } & Modelo burocrático & (a) Recursos públicos directos & \multirow[t]{5}{*}{81.99} \\
\hline & $\begin{array}{l}\text { Generación y asignación de } \\
\text { recursos centralizada }\end{array}$ & Presupuesto incremental negociado & \\
\hline & $\begin{array}{l}\text { Asignación regulada - orientada a } \\
\text { insumos }\end{array}$ & & \\
\hline & Cobertura pública & (b) Financiación indirecta: & \\
\hline & & $\begin{array}{l}\text { Becas, Créditos convencionales, Cero } \\
\text { costos de matrícula }\end{array}$ & \\
\hline \multirow[t]{6}{*}{ Alemania } & Modelo burocrático & (a) Recursos públicos directos & \multirow[t]{6}{*}{70.25} \\
\hline & Sistema federal & Presupuesto incremental negociado & \\
\hline & $\begin{array}{l}\text { Asignación regulada - orientada a } \\
\text { insumos }\end{array}$ & Énfasis en resultados & \\
\hline & Cobertura pública & & \\
\hline & & (b) Financiación indirecta & \\
\hline & & Becas, Bajos costos de matrícula & \\
\hline \multirow[t]{5}{*}{ Inglaterra } & Modelo colegiado & (a) Recursos públicos directos & \multirow[t]{5}{*}{60} \\
\hline & Sistema mixto & Basado en la entrada: fórmulas & \\
\hline & $\begin{array}{l}\text { Asignación regulada - orientada a } \\
\text { insumos }\end{array}$ & & \\
\hline & Cobertura selectiva & (b) Financiación indirecta & \\
\hline & & $\begin{array}{l}\text { Préstamos de ingresos contingente, Altos } \\
\text { costos de matrícula }\end{array}$ & \\
\hline \multirow[t]{5}{*}{ Colombia } & Modelo de mercado & (a) Recursos públicos directos & \multirow[t]{5}{*}{56.43} \\
\hline & $\begin{array}{l}\text { Generación y asignación de } \\
\text { recursos centralizada }\end{array}$ & Presupuesto incremental negociado & \\
\hline & $\begin{array}{l}\text { Asignación regulada - orientada a } \\
\text { insumos }\end{array}$ & & \\
\hline & Cobertura selectiva & (b) Financiación indirecta & \\
\hline & & $\begin{array}{l}\text { Becas, Créditos convencionales, Altos } \\
\text { costos de matrícula }\end{array}$ & \\
\hline
\end{tabular}

Si bien, los países analizados han elegido modelos de financiación que difieren entre sí, principalmente en el rol de los diferentes actores y los mecanismos de ayuda adoptados, conservan algunos elementos en común, como la asignación regulada orientada a insumos y la adopción de préstamos y negociación de recursos. Sin embargo, sus tasas de matriculación en educación terciaria también difieren, lo que aporta a que el debate de la adopción del modelo y el sistema de incentivos elegido continúe. En efecto, una pregunta clave que muchos sistemas educativos se hacen es si el soporte financiero para estudiantes en educación terciaria debería ser en forma de préstamos o becas y subsidios. Parte de la respuesta, se encuentra en que los préstamos pueden incrementar el número de estudiantes beneficiarios de los recursos disponibles y de esta manera el acceso a educación superior, pero resultan menos eficientes que las becas para garantizar el acceso a estudiantes de bajos recursos; de esta manera altos niveles de deuda pueden generar efectos 
negativos si un gran número de estudiantes es incapaz de repagar sus préstamos y si las expectativas de empleo no son suficientes para lograr los pagos.

Para ilustrar el sistema de incentivos de los países de análisis, según datos de la OECD (2019) en Inglaterra más del $90 \%$ de estudiantes recibe únicamente préstamos (y no subsidios o becas) para cubrir el costo de sus estudios. Por su parte, en países donde el costo de matrícula es cercano a 2,000 USD, como en Alemania, menos del $45 \%$ de estudiantes recibe alguna ayuda financiera, siendo la más común becas. En contraste, en Noruega, donde no hay costos de matrícula, alrededor del $75 \%$ de estudiantes reciben apoyo financiero tanto en la forma de préstamos como de becas, destinados a cubrir sus costos de vida. En este contexto de préstamos estudiantiles, un porcentaje importante de estudiantes se encuentra en deuda al momento de la graduación, con montos que en Inglaterra alcanzan los 49,800 USD debido a los altos costos de matrícula; y que, en países nórdicos como Noruega, están sobre los 28,700 USD por los altos costos de vida.

La otra pregunta clave que se hacen los países está asociada a los costos de matrícula y si éstos deberían asumirse de manera compartida o bajo esquemas de gratuidad. Como se mencionó, altos costos de matrícula generan potenciales barreras a la entrada, pero los esquemas de gratuidad también pueden resultar inequitativos, en cuanto las transferencias que hogares pobres estarían realizando a los ricos. Añádase a esto que los costos educativos directos suelen ser altos, principalmente en la educación terciaria, aunque con diferencias para instituciones públicas y privadas. La situación de costos de matrícula para los cuatro países de análisis se muestra en la figura 1.

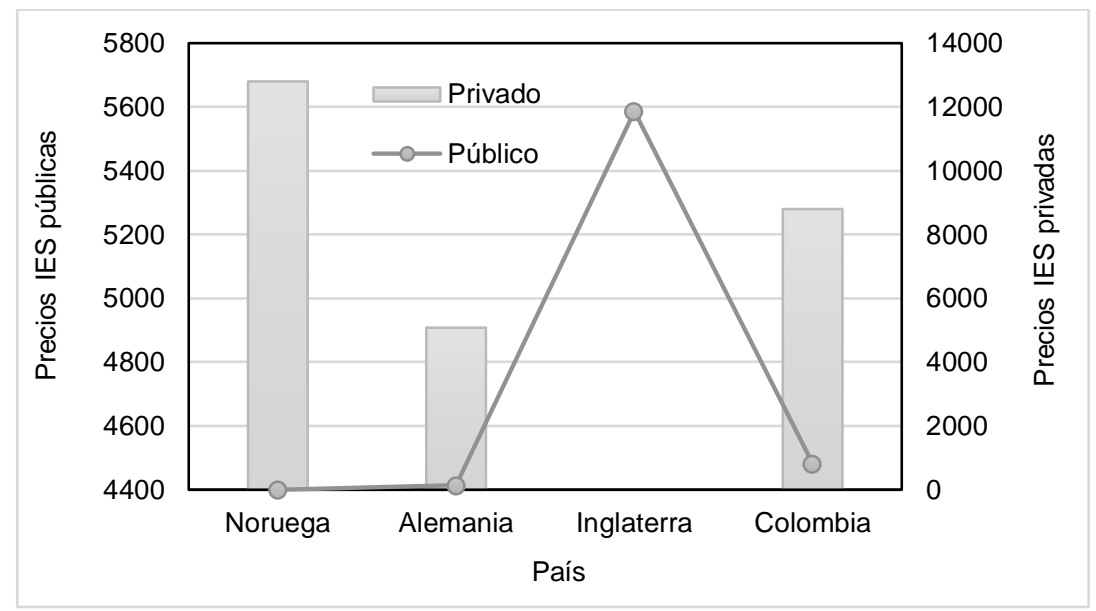

Fig. 1: Costo de matrícula promedio anual en instituciones de educación superior por estudiante, 2016 (USD PPP). (Datos tomados de OECD, 2019)

Pero los costos educativos no son solamente directos, también hay costos indirectos. Los primeros destinados principalmente al pago de matrícula, los segundos asociados a materiales y costos de vida de los estudiantes. De allí, la importancia de diferenciar la fuente de gasto en educación, puesto que muestra la contribución y el impacto que puede tener en el acceso y la provisión del bien educativo. La figura 2 presenta una disminución del gasto público ante el incremento del gasto privado, acorde con la clasificación de países según el modelo de financiación adoptado.

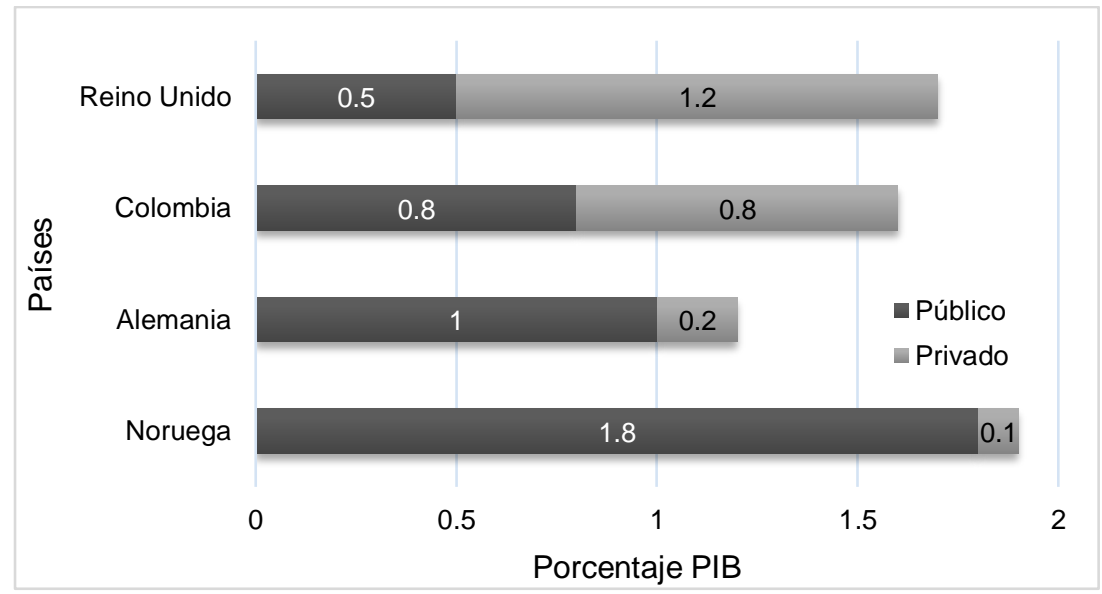

Fig. 2: Gasto público y privado en educación terciaria como porcentaje del PIB, 2016 (Datos tomados de OECD, 2019) 


\section{Análisis Estadístico de Datos Textuales Unidimensional}

Para el análisis estadístico se seleccionaron cuatro países representativos de cada tipo de modelo, a saber: Noruega, modelo 1; Alemania, modelo 2; Inglaterra, modelo 3; y Colombia, modelo 4. La tabla 4 presenta la descripción de los documentos analizados.

Tabla 4. Síntesis documentos de política de financiamiento de educación superior analizados

\begin{tabular}{|c|c|c|c|}
\hline Modelo país & Número de palabras & \% del total & Cantidad de palabras distintas \\
\hline Alemania & 3518 & 49.5 & 552 \\
\hline Colombia & 650 & 9.2 & 209 \\
\hline Noruega & 1370 & 19.3 & 295 \\
\hline Inglaterra & 1564 & 22 & 342 \\
\hline
\end{tabular}

El corpus de análisis fue de 9973 palabras en los cuatro documentos. A este cuerpo se le eliminaron palabras de extensión 1, frecuencia 1 y preposiciones en lengua inglesa, procedimientos de consolidación comunes en el análisis estadístico de datos textuales (Barreto-Galeano et al, 2019; Beaudouin, 2016). Por otra parte, se lematizaron las palabras de acuerdo con las reglas: (a) verbos regulares a la raíz infinitiva y (b) sustantivos y adjetivos en singular masculino. Tras la depuración, el número de palabras analizadas fue de 7444 , y 778 palabras diferentes (10\% del corpus). En la tabla 5 se presentan las 50 primeras palabras llenas (verbos, sustantivos, adjetivos y adverbios) de acuerdo con la frecuencia.

Tabla 5. Cincuenta primeras palabras

\begin{tabular}{|c|l|c|c|l|c|c|c|c|}
\hline Orden & Palabra & Frecuencia & Orden & Palabra & Frecuencia & Orden & Palabra & Frecuencia \\
\hline 1 & Be & 134 & 18 & ministry & 41 & 35 & Support & 26 \\
\hline 2 & university & 133 & 19 & Target & 41 & 36 & management & 25 \\
\hline 3 & education & 108 & 20 & institution & 39 & 37 & More & 25 \\
\hline 4 & Fund & 107 & 21 & System & 38 & 38 & performance & 25 \\
\hline 5 & Is & 96 & 22 & research & 37 & 39 & Fee & 25 \\
\hline 6 & Higher & 83 & 23 & Public & 34 & 40 & Time & 25 \\
\hline 7 & Will & 83 & 24 & Section & 33 & 41 & Berlin & 24 \\
\hline 8 & Are & 82 & 25 & norwegian & 31 & 42 & New & 24 \\
\hline 9 & student & 73 & 26 & May & 30 & 43 & government & 22 \\
\hline 10 & Loan & 58 & 27 & Part & 30 & 44 & Use & 22 \\
\hline 11 & State & 57 & 28 & repayment & 29 & 45 & All & 21 \\
\hline 12 & Year & 50 & 29 & Interest & 28 & 46 & Cost & 21 \\
\hline 13 & Budget & 49 & 30 & Was & 27 & 47 & Were & 21 \\
\hline 14 & Has & 47 & 31 & Act & 26 & 48 & Level & 20 \\
\hline 15 & financial & 45 & 32 & Model & 26 & 49 & Aid & 20 \\
\hline 16 & agreements & 43 & 33 & national & 26 & 50 & Fees & 20 \\
\hline 17 & Have & 42 & 34 & allocation & 26 & & & \\
\hline
\end{tabular}

En primer lugar, en las cincuenta primeras palabras hay una presencia importante del verbo ser/estar (to be). Este resultado es consistente con la alta frecuencia en lengua inglesa de tal verbo irregular. Por otra parte, se encuentran los sustantivos que enuncian a los actores de los modelos (University, State, Students, Institution, Government, etc.), las funciones sustantivas (Education, Research, Support) y los instrumentos de los modelos (Fund, Loan, Budget).Por otra parte, en la tabla 6 se presentan los resultados de los análisis por segmentos, es decir, locuciones conformadas por más de dos palabras con sentido completo. La elección se fundamentó en que las expresiones estuvieran conformadas por dos o más palabras de contenido (sustantivos, adjetivos, verbos, adverbios). Se incluyen las locuciones con más de cinco menciones en los textos. En general, los segmentos repetidos enfatizan en los nombres y acciones de los actores y en los nombres particulares de los instrumentos de financiación. 
Tabla 6. Segmentos repetidos con frecuencia superior a 5.

\begin{tabular}{|c|l|c|}
\hline Orden & \multicolumn{1}{|c|}{ Palabra } & Frecuencia \\
\hline 1 & higher education & 67 \\
\hline 2 & will be & 35 \\
\hline 3 & target agreements & 32 \\
\hline 4 & student financial aid & 20 \\
\hline 5 & education loan & 17 \\
\hline 6 & education institution & 17 \\
\hline 7 & fund allocation & 16 \\
\hline 8 & norwegian state education loan fund & 16 \\
\hline 9 & part time & 14 \\
\hline 10 & regulations concern & 12 \\
\hline 11 & can be & 10 \\
\hline 12 & plan security & 10 \\
\hline 13 & global budget & 10 \\
\hline 14 & science research & 9 \\
\hline 15 & ministry will & 6 \\
\hline
\end{tabular}

Con base en la información suministrada se procedió a caracterizar en términos léxicos los modelos de financiamiento. En la tabla 7 se presentan los vocabularios característicos por presencia, primer bloque de palabras, y ausencia, segundo bloque de palabras. Así, se calcula las asociaciones más y menos allá de lo esperado para cada palabra respecto a los modelos. El reporte usa valores de asociación con valores de probabilidad de error tipo I menores a 0.05 . Las palabras exclusivas del modelo con respecto al corpus total se destacan con un asterisco $\left({ }^{*}\right)$.

Tabla 7. Léxico característico de los modelos.

\begin{tabular}{|c|c|c|c|c|}
\hline Palabras & $\begin{array}{c}\text { Frecuencia } \\
\text { Interna }\end{array}$ & $\begin{array}{c}\text { Frecuencia } \\
\text { Global }\end{array}$ & Valor - test & Probabilidad \\
\hline \multicolumn{5}{|l|}{ Modelo 1} \\
\hline May* & 30 & 30 & 9.636 & 0 \\
\hline Section & 31 & 33 & 9.195 & 0 \\
\hline Act & 25 & 26 & 8.391 & 0 \\
\hline Pursuant* & 20 & 20 & 7.751 & 0 \\
\hline student financial aid* & 20 & 20 & 7.751 & 0 \\
\hline Borrower* & 17 & 17 & 7.093 & 0 \\
\hline norwegian state education loan fund ${ }^{*}$ & 16 & 16 & 6.861 & 0 \\
\hline Applicant* & 15 & 15 & 6.62 & 0 \\
\hline Norwegian* & 15 & 15 & 6.62 & 0 \\
\hline regulations concern* & 12 & 12 & 5,844 & 0 \\
\hline Budget & 1 & 39 & -2.824 & 0.002 \\
\hline State & 1 & 41 & -2.945 & 0.002 \\
\hline Fund & 1 & 75 & -4.631 & 0 \\
\hline \multicolumn{5}{|l|}{ Modelo 2} \\
\hline University & 116 & 133 & 9.162 & 0 \\
\hline target agreements* & 32 & 32 & 6.288 & 0 \\
\hline Management* & 25 & 25 & 5.469 & 0 \\
\hline Performance* $^{\star}$ & 25 & 25 & 5.469 & 0 \\
\hline Berlin* & 24 & 24 & 5.343 & 0 \\
\hline Research & 27 & 28 & 5.239 & 0 \\
\hline higher education & 54 & 67 & 5.145 & 0 \\
\hline
\end{tabular}


Tabla 7 (continuación)

\begin{tabular}{|c|c|c|c|c|}
\hline Palabras & $\begin{array}{l}\text { Frecuencia } \\
\text { Interna }\end{array}$ & $\begin{array}{l}\text { Frecuencia } \\
\text { Global }\end{array}$ & Valor - test & Probabilidad \\
\hline Model & 25 & 26 & 4.986 & 0 \\
\hline Fund & 58 & 75 & 4.839 & 0 \\
\hline Budget & 34 & 39 & 4.781 & 0 \\
\hline Will & 11 & 42 & -2.928 & 0.002 \\
\hline Those & 1 & 14 & -3.086 & 0.001 \\
\hline Benefit & 1 & 15 & -3.266 & 0.001 \\
\hline National & 4 & 26 & -3.423 & 0 \\
\hline Spent & 1 & 16 & -3.439 & 0 \\
\hline \multicolumn{5}{|l|}{ Modelo 3} \\
\hline $\mathrm{Fee}^{*}$ & 25 & 25 & 8.292 & 0 \\
\hline Threshold $^{*}$ & 18 & 18 & 6.99 & 0 \\
\hline Loan & 29 & 41 & 6.527 & 0 \\
\hline part time* & 14 & 14 & 6.079 & 0 \\
\hline Earning $^{*}$ & 11 & 11 & 5.301 & 0 \\
\hline Repayment & 20 & 29 & 5.238 & 0 \\
\hline Student & 29 & 53 & 5.07 & 0 \\
\hline Income & 14 & 17 & 5.063 & 0 \\
\hline Cap* & 10 & 10 & 5.017 & 0 \\
\hline Lower* & 10 & 10 & 5.017 & 0 \\
\hline State & 1 & 41 & -3.316 & 0 \\
\hline University & 6 & 133 & -5.551 & 0 \\
\hline \multicolumn{5}{|l|}{ Modelo 4} \\
\hline $\mathrm{HEls}^{*}$ & 8 & 8 & 5.74 & 0 \\
\hline Billion* & 8 & 8 & 5.74 & 0 \\
\hline Resources & 9 & 13 & 5.052 & 0 \\
\hline Oficial & 7 & 8 & 4.943 & 0 \\
\hline Growth* $^{*}$ & 6 & 6 & 4.864 & 0 \\
\hline Icetex $^{*}$ & 6 & 6 & 4.864 & 0 \\
\hline Public & 13 & 34 & 4.452 & 0 \\
\hline GDP* $^{*}$ & 5 & 5 & 4.366 & 0 \\
\hline Law & 6 & 8 & 4.194 & 0 \\
\hline Transfers $^{*}$ & 4 & 4 & 3.81 & 0 \\
\hline
\end{tabular}

\section{Análisis Estadístico de Datos Textuales Multidimensional}

A partir de la información de individuos (palabras) y categorías (modelos de financiamiento por país), se calculó un análisis factorial que da cuenta del $75.6 \%$ de la varianza a partir de los primeros dos ejes $(x=40.9 \%$ y $y=34.6 \%$ ). En la figura 3 se presentan las 48 palabras llenas o de contenido que más aportan al modelo factorial. La ubicación de las palabras respeta, en general, las coordenadas del modelo; sin embargo, dada la coincidencia de coordenadas, algunas fueron graficadas en distancias cercanas con propósitos ilustrativos. Ahora bien, en ningún caso las palabras se ubicaron por fuera de los cuadrantes resultantes.

El análisis de palabras y distancias sugiere que el eje 1 (izquierda a derecha) representa las características de financiamiento: a la oferta y la demanda, a la demanda o solo a la oferta; en el eje y (arriba abajo) el modelo distingue los costos para los estudiantes, en los puntos más bajos los países con costos más altos para el estudiante - y sus vocabularios característicos - y en los puntos más altos los modelos con costos más bajos - y sus vocabularios característicos -. 


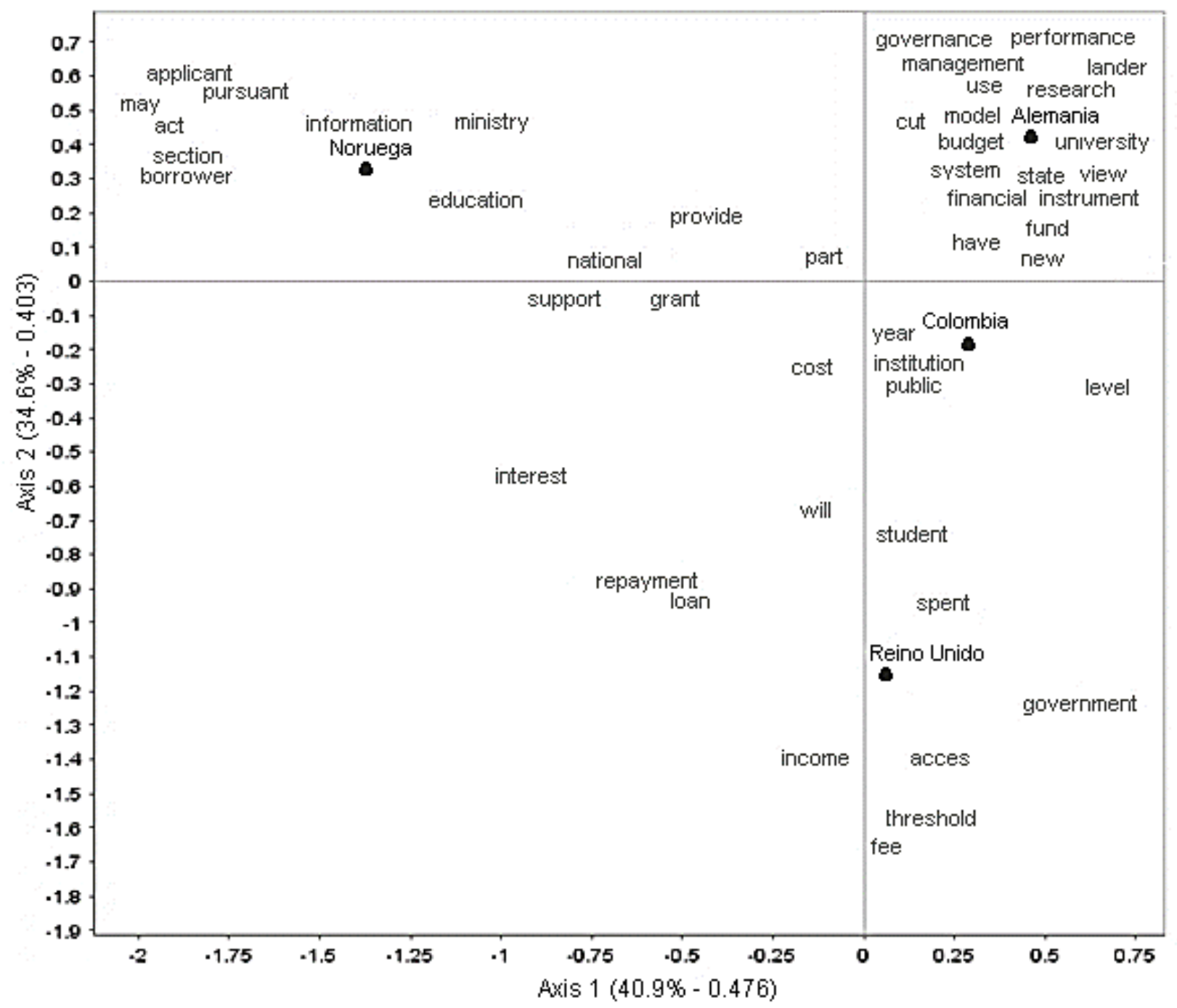

Fig. 3: Análisis factorial del corpus

\section{DISCUSIÓN}

El objetivo de este artículo es contrastar los modelos de financiamiento de la educación superior sugeridos por la OECD (2019) con las políticas de financiamiento que rigen en los diferentes países representativos de cada modelo, esto con el propósito de analizar si existe concordancia entre el planteamiento teórico y el modelo utilizado en la práctica. Para este fin, se recurrió a un análisis estadístico de datos textuales con base en un análisis de correspondencias. Este análisis permite aproximarse a las diferencias y similitudes entre corpus textuales de forma tal que se creen agrupaciones de palabras que permitan caracterizar los textos y así saber cuáles de ellos comparten elementos en común.

En general, la financiación de la educación superior es un conjunto de elecciones públicas y privadas, que responde a preguntas como: ¿quién debería pagar por la educación superior?, ¿en qué proporción?, ¿cómo recaudar los recursos necesarios?, ¿cuántos recursos son suficientes?, ¿cómo realizar su distribución?, entre otras; sustentadas en la valoración de los beneficios educativos individuales y sociales; los cuales fueron promovidos hace ya varias décadas con los postulados de la teoría del capital humano en cabeza de los premios Nobel de economía Gary Becker y Theodore Schultz (Chirat \& Le Chapelain, 2020; Galiakberova, 2019).

Sin embargo, los beneficios sociales no se reducen únicamente a tener sociedades más productivas, sino que la educación también genera innovación y mayores recaudos fiscales (Baum y McPherson, 2011), producción de conocimiento y difusión en la sociedad (Marginson, 2007), mejora en indicadores de salud pública y defensa de derechos humanos o reducción de la criminalidad y participación de estructuras democráticas (McMahon, 2015), por mencionar algunos. Tales externalidades positivas resultan evidentes en las políticas de financiación adoptadas por sistemas de educación superior como el noruego, donde la provisión y el soporte nacionales son protagonistas, junto a la participación de los individuos entendidos como solicitantes de becas o préstamos en caso de requerirse recursos para el sostenimiento, en concordancia con la 
importancia que se otorga al valor social de la educación, resaltado en las características descritas en el modelo 1 del esquema (OECD, 2019).

De igual manera, varios de esos elementos están presentes en el sistema alemán que destaca la importancia de la investigación en las universidades financiadas con fondos estatales, otorgados bajo estructuras de gestión enfocadas en el desempeño. Este resultado coincide con la descripción del modelo 2, donde las instituciones dependen en gran medida de recursos estatales, al valorar los efectos asociados a la producción de conocimiento y su difusión, pero que a su vez pueden ver limitados los recursos ante el pago de tasas de matrícula implementadas en algunos "Lander" junto con la necesidad de tener una mayor y mejor oferta de instrumentos de financiación. Por su parte, el análisis de palabras identificadas para Inglaterra refleja las limitaciones de las altas tasas de matrícula (fee) que potencian las barreras en el acceso, con esquemas de costos compartidos donde el estudiante asume préstamos con tasas de interés y pagos fundamentados en el ingreso futuro (préstamos de ingreso contingente), inherentes al modelo 3 , pero que además se acerca a un sistema de programas de apoyo menos desarrollado de lo esperado (centro derecha-abajo).

Colombia se ubica en la misma línea de sistema de incentivos poco desarrollado, según lo correspondiente al modelo 4, pero hacia el centro (abajo arriba), lo cual se explica por la distribución casi equitativa de la matrícula en instituciones públicas y privadas, que se da con una gran variación en las tasas. Esta situación representa las dificultades en la financiación de los estudiantes, que deben asumir tanto costos de matrícula como costos de sostenimiento; en un contexto en el que el discurso de la política educativa resalta elementos de gasto público, que no logran cumplir con los criterios de eficiencia y equidad deseados.

\section{CONCLUSIONES}

Los principales hallazgos corroboran los elementos propios de los modelos de financiación de educación superior analizado a la luz del esquema utilizado por la OCDE (2019), frente al análisis estadístico de datos textuales en los cuatro países seleccionados, con algunas diferencias. En primer lugar, el sistema de incentivos a estudiantes de Inglaterra resulta menos desarrollado de lo esperado, lo que sugiere una revisión de su política de préstamos. En segundo lugar, la gran variación en las tasas de matrícula provenientes de una oferta mixta de instituciones en Colombia sugiere una política de gasto público que no alcanza a cubrir a la mayoría de la población y la enfrenta a grandes dificultades en la financiación de sus estudios. Finalmente, Noruega y Alemania representan claramente el modelo en que la sociedad otorga una gran importancia a la educación y la investigación financiadas con recursos públicos, con variaciones en las tasas de matrícula y los programas de apoyo a los estudiantes; propias de los modelos 1 y 2.

\section{REFERENCIAS}

Adam, E., 'Governments base performance-based funding on global rankings indicators': A global trend in higher education finance or a global rankings literature fiction? A comparative analysis of four performance-based funding programs, https://doi.org/10.1016/j.ijedudev.2020.102197, Int. J. Educ. Dev., 1-11 (2020)

Araneda-Girriman, C. A., Gairín-Sallán, J. y Pedraja-Rejas, L. M., La autonomía en la educación superior: reflexiones desde los actores en el contexto del financiamiento por desempeño en Chile, http://dx.doi.org/10.4067/S071850062018000400065, Form. Univ., 11(4), 65-74 (2018)

Araneda-Girriman, C. A., y Pedraja-Rejas, L. M., Financiamiento por desempeño en Chile: Análisis conceptual de un instrumento para la educación superior, http://dx.doi.org/10.4067/S0718-50062016000300009, Form. Univ., 9(3), 75-86 (2016)

Barreto-Galeano, M. I., Medina-Arboleda, I. F. y otros cuatro autores, Rhetoric, political ideology and the peace process in Colombia: A Twitter® Analysis, https://doi.org/10.1080/1057610X.2019.1615256, Analysis. Stud. Confl. Terror., 1-18 (2019)

Beaudouin, V., Statistical analysis of textual data: Benzécri and the French school of data analysis. Glottometrics, 33, 56$72(2016)$

Chapman, B. y Doris, A., Modelling higher education financing reform for Ireland, https://doi.org/10.1016/j.econedurev.2018.06.002, Econ. Educ. Rev., 71, 109-119 (2019)

Chirat, A., y Le Chapelain, C., Economic analysis of education in post-war America: new insights from Theodore Schultz and John Kenneth Galbraith, https://doi.org/10.1017/S1053837218000779, J Hist Econ Thought., 42(1), 61-78. (2020)

Consejo Nacional de Educación Superior (CESU), Acuerdo por lo superior 2034. Propuesta de política pública para la excelencia de la educación superior en Colombia en el escenario de la paz. Colombia. (2013)

De Donder, P. y Martínez-Mora, F., The political economy of higher education admission standards and participation gap, https://doi.org/10.1016/j.jpubeco.2017.07.004, J. Public. Econ., 154, 1-9 (2017)

Del Rey, E. y López-García, M., On government-created credit markets for education and endogenous growth, https://doi.org/10.1016/j.econmod.2019.12.016, Econ. Model., 92, 170-179 (2020) 
Galiakberova, A Conceptual Analysis of Education Role in Economics: The Human Capital Theory. doi: 10.7596/taksad.v8i3.2256, J. Hist. Cult. Art. Res., 8(3), 410-421 (2019)

Gaxiola Romero J. C., Gaxiola Villa E., Corral Frías N. S. y Escobedo Hernández P., Ambiente de aprendizaje positivo, compromiso académico y aprendizaje autorregulado en bachilleres, https://doi.org/10.14718/ACP.2020.23.2.11, Acta Colom. Psicol., 23(2), 267-288 (2020)

Gentzkow, M., Kelly, B. y Taddy, M., Text as data, https://doi.org/10.1257/jel.20181020, J Econ Lit., 57(3), 535-74 (2019)

Gershenfeld, S., Zhan, M. y Hood, D. W., The impact of a promise: A loan replacement grant, low-income students, and college graduation, doi: 10.1353/rhe.2019.0030, Rev. High. Educ., 42(3), 1073-1100 (2019)

Haupt, A., The evolution of public spending on higher education in a democracy, https://doi.org/10.1016/j.ejpoleco.2012.05.003, Eur. J. Polit. Econ, 28, 557-573, (2012)

Johnstone, D. B., The economics and politics of cost sharing in higher education: comparative perspectives, https://doi.org/10.1016/j.econedurev.2003.09.004, Econ. Educ. Rev., 23(4), 403-410 (2004)

Jongbloed, D. B., Marketisation in higher education, Clark's triangle and the essential ingredients of markets, https://doi.org/10.1111/1468-2273.00238, High. Educ. Q., 57(2), 110-135 (2003)

Marginson, S., The public/private divide in higher education: a global revision, https://doi.org/10.1007/s10734-005-8230y., High. Educ., 53, 307-333 (2007)

Marginson, S., Public/private in higher education: a synthesis of economic and political approaches, doi: 10.1080/03075079.2016.1168797, Stud. High. Educ., 43(2), 322-337 (2018)

McMahon, W., Financing Education for the Public Good: A New Strategy, J. Educ. Financ, 40(4), ISSN: 1944-6470, 414437, (2015)

Ministry of Education and Research of Norway, Department of Higher Education and Research Institutions. Student Financial Aid Act. Noruega. (2005)

Organization for Cooperation and Economic Development -OECD-. Funding systems and their effects on higher education systems. Alemania. (2006)

Organization for Cooperation and Economic Development -OECD- Education at a Glance 2018: OECD Indicators. Paris: OECD Publishing. (2018)

Organization for Cooperation and Economic Development -OECD-. Education at a Glance 2019: OECD Indicators. Paris: OECD Publishing. https://doi.org/10.1787/f8d7880d-en (2019)

Parliament United Kingdom - House of Commons Library, Research Briefings Changes to higher education funding and student support from 2012/13. Inglaterra. (2013)

Río-Ruíz, M.A., Jiménez-Rodrigo, M.L. y Caro-Cabrera, M.J., The shifting financial aid system in Spanish University: grantrecipients' experiences and strategies, https://doi.org/10.1080/17508487.2014.969286, Crit. Stud. Educ., 56(3), 332-350 (2015)

Romero-Pérez, I., Alarcón-Vásquez, Y., García-Jiménez, R., Lexicometría: enfoque aplicado a la redefinición de conceptos e identificación de unidades temáticas, http://dx.doi.org/10.5195/biblios.2018.466, J. Libr. Inf. Sci., (71), 68-80 (2018)

Sanyal, B.C. y Johnstone, D.B., International trends in the public and private financing of higher education, https://doi.org/10.1007/s11125-011-9180-z, Prospects, 41, 157-175 (2011)

Unesco. Inscripción escolar, nivel terciario (\% bruto). (2020)

Van-Long, N., Financing higher education in an imperfect world, https://doi.org/10.1016/j.econedurev.2018.06.004, Econ. Educ. Rev., 71, 23-31 (2019) 